\title{
Learning behavior in rat offspring after in utero and lactational exposure to either TCDD or PCB126
}

\author{
Rieko Hojo · Masaki Kakeyama • Yoshika Kurokawa • \\ Yasunobu Aoki · Junzo Yonemoto · Chiharu Tohyama
}

Received: 29 May 2007/ Accepted: 4 January 2008/Published online: 29 March 2008

(c) The Japanese Society for Hygiene 2008

\begin{abstract}
Objectives We studied and compared the possible effects of in utero and lactational exposure to $2,3,7$, 8-tetrachlorodibenzo- $p$-dioxin (TCDD) or 3, 3', 4, 4', 5-pentachlorobiphenyl (PCB126) on learning behavior in offspring.

Methods Pregnant Long-Evans Hooded rats were administered either TCDD $(50,200$, or $800 \mathrm{ng} / \mathrm{kg}$ ) or PCB126 (500, 2,000 or 8,000 ng/kg) on gestational day 15 . A procedure of schedule-controlled operant behavior was applied to examine learning behavior in the male and female offspring at 11 weeks of age for 30 days. Three indices, namely, response rates in a fixed ratio (FR) and in a differential reinforcement of low rates (DRL), and reward rate in the DRL component in multiple FR 20 DRL $20 \mathrm{~s}$ (mult-FR 20 DRL 20-s) test sessions, were used for the evaluation of learning behavior.

Results Toxic effects on learning behavior in male and female pups following in utero and lactational exposure to TCDD or PCB126 were observed mainly in the FR learning component. However, no linear dose-dependent
\end{abstract}

R. Hojo · Y. Kurokawa · Y. Aoki · J. Yonemoto · C. Tohyama

Research Center for Environmental Risk,

National Institute for Environmental Studies,

Tsukuba 305-8506, Japan

M. Kakeyama · C. Tohyama $(\square)$

Laboratory of Environmental Health Sciences,

Center for Disease Biology and Integrative Medicine,

Graduate School of Medicine, The University of Tokyo,

7-3-1 Hongo, Bunkyo-ku, Tokyo 113-0033, Japan

e-mail: mtohyama@mail.ecc.u-tokyo.ac.jp effects of either of the two compounds were observed for the above three indices. The response rates of animals in the low-dose TCDD and PCB126 groups decreased and those in medium-dose TCDD and PCB126 groups appeared to induce hyperactive behavior. The high dose of PCB126 appeared to have a distinct toxicity from that of TCDD in terms of the acquisition of learning behavior.

Conclusions Toxicities of PCB126 and TCDD in learning behavior might be similar to each other and the current toxic equivalency factor (TEF) of 0.1 for PCB126 can be considered to be appropriate for this endpoint.

Keywords Learning - Operant behavior · PCB126 . Rat $\cdot$ TCDD

$\begin{array}{ll}\text { Abbreviations } \\ \text { AhR } & \text { Aryl hydrocarbon receptor } \\ \text { CRF } & \text { Continuous reinforcement } \\ \text { CYP1A1 } & \text { Cytochrome P450; family 1, subfamily A, } \\ & \text { polypeptide 1 } \\ \text { DRL } & \text { Differential reinforcement of low rates } \\ \text { FI } & \text { Fixed interval } \\ \text { FR } & \text { Fixed ratio } \\ \text { GD } & \text { Gestational day } \\ \text { Mult } & \text { Multiple reinforcement schedule } \\ \text { PCB126 } & 3,3^{\prime}, 4,4^{\prime}, 5 \text {-Pentachlorobiphenyl } \\ \text { PND } & \text { Postnatal day } \\ \text { REP } & \text { Relative potency } \\ \text { RR } & \text { Response rate in FR } \\ \text { RR } & \text { Response rate in DRL } \\ \text { RR }_{\text {DRL }} & \text { Reward rate in DRL } \\ \text { SCOB } & \text { Schedule controlled operant behavior } \\ \text { TCDD } & 2,3,7,8 \text {-Tetrachlorodibenzo- } p \text {-dioxin } \\ \text { TEF } & \text { Toxicity equivalency factor }\end{array}$




\section{Introduction}

Polychlorinated dibenzodioxins (PCDDs), polychlorinated dibenzofurans (PCDFs) and polychlorinated biphenyls (PCBs) are a group of persistent environmental chemicals. Among the more than 400 congeners belonging to this group, 29 are classified as dioxins and related compounds because they have been shown to exert several toxic responses similar to those of 2,3,7,8-tetrachloro- $p$-dibenzodioxin (TCDD), the prototype of these compounds [1]. Among the PCB congeners, both non- and mono-ortho PCB congeners have toxicological characteristics similar to those of TCDD via the aryl hydrocarbon receptor-mediated mechanism; these are often called dioxin-like PCBs [2]. For practical risk assessment purposes, the toxic equivalents (TEQ) is used to evaluate the total dioxin toxicity of various congeners to which humans are exposed through food and environmental media. A degree of toxicities of these congeners are expressed as toxic equivalent factors (TEF) and range from 1.0 to 0.00001 , with the TEF value 1.0 given to the most toxic congener, TCDD, as the standard $[1,3]$. The TEF value is a composite one that is derived, on the basis of a so-called expert assessment, from the relative potency (REP) obtained from various experiments, both in vivo and in vitro.

Previous in vivo studies showed that a low TCDD dose that did not affect the dams did disrupt the offspring's learning behavior when the offspring was challenged by various behavioral tests, such as matching-to-sample learning tasks in monkeys [19], a cued alternation task in rats [20] and a schedule-controlled operant behavior procedure in rats [21]. In a cued delayed alternation procedure [20], adult male and female Long-Evans rats, whose dams had been exposed to a single dose of $180 \mathrm{ng} / \mathrm{kg}$ of TCDD on gestation day (GD) 15, showed significantly less accurate learning behavior and committed more errors by responding during delay intervals. The results of this study suggested that TCDD interfered with the development of attention processes, impaired response inhibition and/or promoted response inhibition despite the presence of cues, indicating changes in reinforcement contingencies. In another study, rats that were born to dams that received a single dose of $0,20,60$, or $180 \mathrm{ng} / \mathrm{kg}$ of TCDD on GD 8 were examined for operant learning behavior during the differential reinforcement of low rate (DRL) component of a multiple fixed ratio (FR) DRL reinforcement schedule [21]. These results indicated that levels of less than $180 \mathrm{ng} /$ $\mathrm{kg}$ of maternal TCDD exposure affected the learning behavior of offspring even after the offspring developed.

The TEF methodology was developed nearly two decades ago [4, 5], and the REP dataset has been developed further by scientists at the Karolinska Institute in Stockholm, Sweden. The historical changes in this methodology were clearly elaborated by Haws et al. [6]. The most widely used TEF scheme is that compiled by a World Health Organization (WHO) expert panel in 1997, widely recognized as $\mathrm{WHO}^{\mathrm{TEF}} \mathrm{TE}_{98}$ [3]. The panel recommended TEFs for 29 congeners, including 17 laterally substituted PCDDs/PCDFs, four mono-ortho PCBs, and eight nonortho PCBs in this TEF scheme [3]. This TEF scheme was developed on the basis of the following four basic criteria. The compound must (1) show a structural relationship to the PCDDs and PCDFs, (2) bind to the aryl hydrocarbon receptor (AhR) receptor, (3) elicit AhR-receptor-mediated biochemical and toxic responses and (4) be persistent and accumulate in the food chain. The TEF scheme is a pragmatic concept and has been utilized for risk assessment and management purposes, but the REP dataset used to derive the TEF values has some innate limitations. Haws et al. [6] revised the REP dataset for dioxins and related compounds by focusing on the recompilation of the dataset used for the development of the $\mathrm{WHO} \mathrm{TEF}_{98}$ and the enhancement of quantitative evaluation and the transparency of REPs of various experimental data in vivo and in vitro. In the newly proposed database, numerous data from the previous version of the REP dataset were excluded, but these were offset by the addition of several new REP values from studies published since 1977.

In terms of the toxicities of dioxin-like PCBs, experimental evidence has shown that 3, 3', 4, 4', 5pentachlorobiphenyl (PCB126) is the most toxic compound among 209 congeners of PCBs that consist of non-orthoPCBs, mono-ortho-PCBs and di-ortho-PCBs. Based on various experimental data from the REP of PCB126, 86 REPs obtained in in vivo experiments are included in the newly revised dataset [6]. The endpoints used as the bases for listing REPs include alterations in body weight, thymus weight, vitamin A metabolism, porphyrin metabolism, immune functions and presence of cleft palate as well as enzyme induction (CYP1A1 and UDP-glucuronosyltransferase). Nearly all of these data were obtained from adult rodent experiments. As yet, no data on behavioral toxicity are included in the database except those from our laboratory. These data were preliminary and were presented as part of report at the International Symposium on Halogenated Environmental Organic Pollutants and Persistent Organic Pollutants, Berlin, 2004 [7] and are presented here in this complete report.

A number of previous studies have examined the effects of PCB126 exposure to dams on the behavior and learning behavior in offspring rodents [8-14], although data from such studies are not included in the REP dataset, presumably because they do not meet the criteria for the revised the dataset [6]. The endpoints used in these studies were learning behavior, such as spatial learning, visual discrimination and transitional behavior, and open-field 
locomotor activity of offspring born to dams that were exposed to PCB126. In the study reported here, we selected and studied neurobehavioral functions using the so-called schedule-controlled operant behavior (SCOB) procedure. This procedure has been widely used in the fields of behavioral pharmacology and toxicology because it is highly sensitive in detecting a subtle alterations (see reviews in [15-18]).

To study the possible effects of in utero and lactational exposure to toxicants on offspring, it is essential to use this dosing paradigm. In several studies, researchers who examined the effects of TCDD exposure during pregnancy or lactational period on offspring reported that the doses of TCDD used in their study were sufficiently low not to affect dams, but sufficiently high to disrupt offspring's learning behavior, as evaluated using various behavioral tests, such as matching-to-sample learning tasks in monkeys [19], a cued alternation task in rats [20] and a schedule-controlled operant behavior procedure in rats [21]. Therefore, we selected a dose of PCB126 and TCDD that presumably does not affect even the body weight of dams. Furthermore, we utilized the in utero and lactational exposure protocol that has been used to study developmental toxicity in terms of critical windows during pregnancy in rodents [22].

\section{Material and methods}

\section{Chemicals}

TCDD was purchased from Cambridge Isotope Laboratory (Andover, MA, USA) with a purity of more than $99.9 \%$. PCB126 was purchased from AccuStandard (New Haven, CT, USA) with a purity of more than $99.5 \% . N$-nonane and corn oil was purchased from Sigma (St Louis, MO, USA).

\section{Animals}

Female and male Long-Evans hooded rats, all 6 weeks old, were purchased from Charles River (Chicago, IL, USA). Female and male rats were housed individually in polycarbonate cages in a controlled environment $\left(24 \pm 1^{\circ} \mathrm{C}\right.$, $45 \pm 5 \%$ humidity, 12/12-h light/dark photoperiod) in a barrier facility provided with filtered air. The daily diet consisted of Daily Diet Rodent Chow (Cat. Code: CA-1; Japan CLEA, Tokyo, Japan); both this Chow and tap water were provided ad libitum. After 2 weeks of acclimatization to the vivarium quarters, a female rat was placed with a male overnight (approximately from 4:00 p.m. to 9:00 a.m.) in a wire cage. Gestation day 0 was designated as the day when the virginal plug was detected the following morning, and pregnant rats were individually kept in a polycarbonate cage. A total of 51 pregnant rats were used for the subsequent experiments. The animals used in the experiments were handled with humane care in accordance with the guidelines on laboratory animal experiments at the National Institute for Environmental Studies.

\section{Administration of TCDD and PCB126}

TCDD and PCB126 were dissolved in corn oil containing $0.5 \%$-nonane, and on GD 15 pregnant rats were administered either the TCDD or PCB126 solution by gavage $(2.5 \mathrm{ml} / \mathrm{kg})$ or only the corn oil containing $0.5 \% n$-nonane. The animals were assigned to seven groups: three groups of pregnant rats exposed to TCDD [low (50 ng/kg), medium (200 ng/kg) and high (800 ng/kg) doses], three groups of pregnant rats exposed to PCB126 [low (500 ng/kg), medium $(2,000 \mathrm{ng} / \mathrm{kg})$ and high $(8,000 \mathrm{ng} / \mathrm{kg})$ doses, a TEF value of 0.1 [1] was adopted] and one vehicle group.

\section{Litters}

Postnatal day (PND) 0 was designated as the first day when the delivery of offspring was observed by 8:30 a.m.; the gestational length, number of live offspring, sex ratio at birth and gross appearance of the offspring were examined on PND 1. The body weight of offspring was recorded on PNDs 4, 8, 12, 16, and 20. At 10 weeks of age, one male and one female offspring were randomly selected from each litter to form groups used for experiments. Each group consisted of six to eight offspring of either sex. In the behavioral tests, one male and female from each litter were used. The standard weights of the male and female offspring at 10 weeks of age are 290-330 and 200-255 g, respectively, according to the breeder's background data. To maintain $80-85 \%$ of their free-feeding weight on the basis of the standard body weights, the daily diets were restricted to 20 and $16 \mathrm{~g}$ per day for male and female offspring, respectively, after 10 weeks of age. Through this restriction in the amount of the daily diet, food (see Behavioral tests for details) used in the behavioral test was thought to be an effective reward for the animals. Animals had ad libitum access to water.

\section{Behavioral tests}

The behavioral tests were conducted in operant conditioning chambers $[500 \mathrm{~mm}$ (width) $\times 280 \mathrm{~mm}$ (depth) $\times 325 \mathrm{~mm}$ (height), model MSK-002R; Muromachi Kikai, Tokyo, Japan] that were designed for rats. Two levers were mounted on the front wall of the chamber, $80 \mathrm{~mm}$ above the floor, $120 \mathrm{~mm}$ apart from each other. A recessed feeder receptacle was mounted between the levers, $40 \mathrm{~mm}$ above the floor. The operant chamber was 
housed inside a double-walled, insulated sound-attenuating chamber with a fan for ventilation (ICM Implement, Tsukuba, Japan).

Laboratory rodent chow (45 mg; Noyes Precision Food Pellets; Rodent Diet, P.J. Noyes Co., Lancaster, NH, USA) was used as a food reward for the behavioral tests. When a pellet was delivered, a feeder light located on the ceiling inside the feeder was turned on for $0.5 \mathrm{~s}$. A house light was mounted in the center of the front wall $15 \mathrm{~mm}$ below the ceiling, and this was turned on at the beginning of an experiment. Task operation and data acquisition were carried out with a programming package ComPACT (Muromachi Kikai, Tokyo, Japan) running on Windows XP. Time resolution for data acquisition was $10 \mathrm{~ms}$.

All schedules of the SCOB procedure were designed and implemented as described in a previous report [21]. In brief, each animal was assigned to perform one session per day, five sessions per week and 42 sessions in 9 weeks. Each complete set of 42 sessions consisted of 12 training sessions (first 12 sessions) followed by 30 test sessions. Throughout all training and test sessions, a food reward was provided only for the lever press response of animals.

Training was initiated at 10 weeks of age, and four operant schedules were assigned to the animals:

1) Continuous reinforcement (CRF) schedule; in this schedule, a food pellet was served whenever a rat pressed the lever once. This schedule was used for rats to learn the lever-press behavior and consisted of four sessions.

2) Fixed ratio (FR) schedule; after the CRF schedule, the FR schedule was started as FR3, which means that the third-lever press was reinforced by a food pellet. Animals were then exposed to FR5 for one session and FR10 for two sessions. The house light, used as a discrimination stimulus for the FR schedule, was turned on during the schedule.

3) Differential reinforcement of low rates (DRL) schedule. This schedule reinforced a consecutive series of lever presses during a specified time interval: the number in the DRL indicates the duration that animals had to wait before their next response (lever pressing). If the animal pressed the lever within the duration, the clock counter was reset to 0 . In the training for the DRL schedule, DRL5s and DRL10s for one and two sessions, respectively were used. The house light was turned off during the schedule.

4) As the final training, a multiple FR10 DRL10s (mult FR10 DRL10-s) schedule was conducted for two sessions. There were two components (2-min FR10 and 5-min DRL), which were alternately repeated seven times, in the mult-FR10 DRL10-s schedule.
In the training for the CRF, the FR3 and FR5 schedules lasted $30 \mathrm{~min}$ or until the animal acquired 50 pellets. The sessions for the FR10, DRL5s, DRL10s and mult-FR10 DRL10-s schedule lasted $60 \mathrm{~min}$ or until the animals acquired 100 pellets. As the test protocol, a mult-FR 20 DRL 20-s schedule was started at 3 months of age after the training described above had been accomplished. Each session was conducted once a day for 30 days, for a total of 49 min or until the animals acquired 50 pellets. In order to evaluate learning behavior, we used FR and DRL schedules, both of which are the two components in the multiple schedule of operant behavior and have been widely used in behavioral teratology studies $[7,21]$. The FR schedule can be used as an indicator of reflect motor activity, attention and hyper/hypo activity. In contrast, the DRL schedule is used to evaluate time estimation and the waiting responding behavior, namely, the responding suppression of animals.

\section{Data analysis}

Differences in means among the groups were analyzed for gestational length, number of live offspring and sex ratio at birth among groups by one-way analysis of variance (ANOVA). One-way ANOVA with repeated measures, followed by Turkey's test as post hoc analysis, was adopted for comparing the body weights of the dams and pups and for comparing three indices-response rate, defined as the number of lever presses per minute in FR $\left(R_{\mathrm{FR}}\right)$, response rate in DRL $\left(\mathrm{RR}_{\mathrm{DRL}}\right)$ and reward rate, the number of food rewards per minute in DRL $\left(\mathrm{rR}_{\mathrm{DRL}}\right)$-in the mult-FR 20 DRL 20-s test sessions. Test sessions were divided into three ten-session blocks (phases 1, 2 and 3). Because all three indices had a normal distribution, they were subjected to logarithmic transformation. For repeated measures ANOVA, three between-subject factors, namely chemical (TCDD and PCB126), dose (control, low, medium, and high) and sex, and one within-subject factor, namely, phase (1, 2 and 3), were set. Each phase consisted of a ten-sessionblock, and this was used to assess the change in the behavioral tendency at the beginning, middle or end of the behavioral test. In addition, to estimate the difference in the learning behavior among animal group, means of response rate and reward rate of the last five sessions, which were considered to be a steady state of the learning task, in each of $\mathrm{RR}_{\mathrm{FR}}$ and $\mathrm{RR}_{\mathrm{DRL}}$ were analyzed by one-way ANOVA. All statistical analyses were performed using the SPSS program version 10.0 (SPSS Japan, Tokyo, Japan). The probability level required for significance was set at $p<0.05$. 


\section{Results}

Pregnancy and growth

Neither TCDD nor PCB126 at low, medium and high doses affected the gestational length, body weight of dams, litter size and sex ratio at birth in comparison with the vehicleadministered control dams (Table 1).

During the preweaning period, neither TCDD nor PCB126 exposure to dams affected the body weights of either the male and female pups, suggesting that no marked toxicity was relevant to the possible effects of either compound on the learning behavior of the offspring (Table 2).
Behavioral tests

Repeated measures ANOVA was used to evaluate the possible effects of dose, chemical (TCDD or PCB126), sex, phase and dose-chemical interaction in the response rate of male and female offspring in the FR component (Fig. 1).

In the FR component, the medium-dose group for TCDD (200 ng/kg) showed a significantly higher response rate $(p=0.005)$ than the other dose groups in terms of the female offspring. A similar response pattern was observed for male offspring, but this was not statistically significant. Similarly, the medium-dose group for PCB126 (2,000 ng/ $\mathrm{kg}$ ) tended to show a higher response rate than other groups in terms of both male and female offspring. The response

Table 1 Profiles of pregnancy in the groups of dams exposed to 2, 3, 7, 8-tetrachlorodibenzo- $p$-dioxin (TCDD) or 3, 3', 4, 4', 5-pentachlorobiphenyl (PCB126)

\begin{tabular}{|c|c|c|c|c|c|c|c|}
\hline \multirow[t]{2}{*}{ Groups $^{\mathrm{a}}$} & \multirow{2}{*}{$\begin{array}{l}\text { Number } \\
\text { of dams }\end{array}$} & \multirow{2}{*}{$\begin{array}{l}\text { Length of } \\
\text { pregnancy } \\
\text { (days) }\end{array}$} & \multirow{2}{*}{$\begin{array}{l}\text { Body weight } \\
\text { of dams }(\mathrm{g})\end{array}$} & & \multicolumn{2}{|c|}{ Number of pups } & \multirow[t]{2}{*}{ Sex ratio ${ }^{b}$} \\
\hline & & & & & Male & Female & \\
\hline Control & 7 & $21.8 \pm 0.5$ & $333.8 \pm 17.2$ & $15.4 \pm 1.1$ & $6.6 \pm 2.0$ & $8.8 \pm 1.9$ & $0.83 \pm 0.39$ \\
\hline TCDD 50 & 8 & $21.6 \pm 0.5$ & $331.6 \pm 21.1$ & $11.0 \pm 3.7$ & $4.9 \pm 2.1$ & $6.1 \pm 3.3$ & $1.15 \pm 0.75$ \\
\hline TCDD 200 & 6 & $21.2 \pm 0.4$ & $341.8 \pm 31.8$ & $10.8 \pm 4.5$ & $5.2 \pm 3.5$ & $5.7 \pm 1.8$ & $0.88 \pm 0.72$ \\
\hline TCDD 800 & 6 & $21.5 \pm 0.5$ & $348.8 \pm 25.6$ & $13.0 \pm 1.9$ & $7.2 \pm 1.6$ & $5.8 \pm 2.4$ & $1.56 \pm 1.05$ \\
\hline PCB126 500 & 8 & $21.7 \pm 0.5$ & $327.6 \pm 44.4$ & $14.3 \pm 1.3$ & $7.9 \pm 1.5$ & $6.4 \pm 1.4$ & $1.18 \pm 0.43$ \\
\hline PCB126 2000 & 8 & $21.3 \pm 0.7$ & $337.0 \pm 31.7$ & $13.8 \pm 2.1$ & $7.8 \pm 2.1$ & $6.0 \pm 1.9$ & $1.19 \pm 1.41$ \\
\hline PCB126 8000 & 8 & $21.4 \pm 0.5$ & $343.4 \pm 35.6$ & $14.1 \pm 2.3$ & $7.6 \pm 1.8$ & $6.5 \pm 1.7$ & $1.17 \pm 0.34$ \\
\hline
\end{tabular}

Values are given as the mean \pm standard error of the mean (SEM)

${ }^{a}$ Pregnant Long-Evans hooded rats were administered either TCDD or PCB126 on gestational day 15. Groups of low, medium and high doses of TCDD and PCB126 were 50, 200 and $800 \mathrm{ng} / \mathrm{kg}$ of TCDD and 500, 2000 and 8,000 ng/kg of PCB126, respectively

b Sex ratio is given at $\delta / q \pm$ standard deviation

Table 2 Body weights of male and female pups born to dams exposed in utero and lactational exposure to TCDD or PCB126
Values are given as the mean \pm SEM

PND, Postnatal day

${ }^{\text {a }} \mathrm{ng} / \mathrm{kg}$

\begin{tabular}{llcllll}
\hline Group $^{\mathrm{a}}$ & Sex & \multicolumn{5}{l}{ Body weight of pups (g) } \\
\cline { 3 - 7 } & & PND 4 & PND 8 & PND 12 & PND 16 & PND 20 \\
\hline Control & Male & $9.4 \pm 0.7$ & $18.2 \pm 1.4$ & $28.3 \pm 2.6$ & $38.5 \pm 4.7$ & $50.1 \pm 4.3$ \\
& Female & $9.2 \pm 0.9$ & $17.6 \pm 1.2$ & $27.6 \pm 2.6$ & $37.2 \pm 3.8$ & $48.6 \pm 3.5$ \\
TCDD 50 & Male & $10.6 \pm 1.4$ & $17.1 \pm 2.2$ & $26.7 \pm 2.9$ & $36.3 \pm 3.1$ & $48.4 \pm 4.7$ \\
& Female & $9.6 \pm 1.1$ & $16.1 \pm 1.7$ & $25.2 \pm 2.3$ & $35.0 \pm 3.1$ & $46.4 \pm 3.8$ \\
TCDD 200 & Male & $9.8 \pm 1.3$ & $15.5 \pm 2.4$ & $26.5 \pm 3.3$ & $35.6 \pm 3.4$ & $47.1 \pm 5.4$ \\
& Female & $9.9 \pm 1.6$ & $15.7 \pm 2.1$ & $26.7 \pm 3.1$ & $35.3 \pm 3.6$ & $46.9 \pm 5.2$ \\
TCDD 800 & Male & $9.7 \pm 0.9$ & $16.7 \pm 3.5$ & $26.8 \pm 3.4$ & $36.1 \pm 3.8$ & $47.2 \pm 5.0$ \\
& Female & $9.2 \pm 1.1$ & $15.9 \pm 2.8$ & $26.2 \pm 2.8$ & $35.5 \pm 2.9$ & $46.2 \pm 3.9$ \\
PCB126 500 & Male & $9.5 \pm 0.8$ & $18.1 \pm 1.6$ & $27.4 \pm 2.3$ & $37.9 \pm 3.3$ & $48.8 \pm 3.6$ \\
& Female & $8.8 \pm 0.7$ & $16.7 \pm 1.3$ & $25.4 \pm 2.1$ & $35.6 \pm 3.2$ & $45.6 \pm 3.7$ \\
PCB126 2000 & Male & $8.9 \pm 1.0$ & $16.4 \pm 2.5$ & $25.4 \pm 4.5$ & $34.9 \pm 4.6$ & $47.0 \pm 5.4$ \\
& Female & $8.3 \pm 1.2$ & $15.4 \pm 2.6$ & $23.9 \pm 4.2$ & $33.3 \pm 4.5$ & $43.9 \pm 5.4$ \\
PCB126 8000 & Male & $9.2 \pm 1.0$ & $15.4 \pm 1.9$ & $25.5 \pm 2.8$ & $33.5 \pm 3.3$ & $44.5 \pm 4.8$ \\
& Female & $8.8 \pm 0.8$ & $14.7 \pm 1.9$ & $24.3 \pm 2.6$ & $31.8 \pm 3.0$ & $42.6 \pm 5.1$ \\
\hline
\end{tabular}




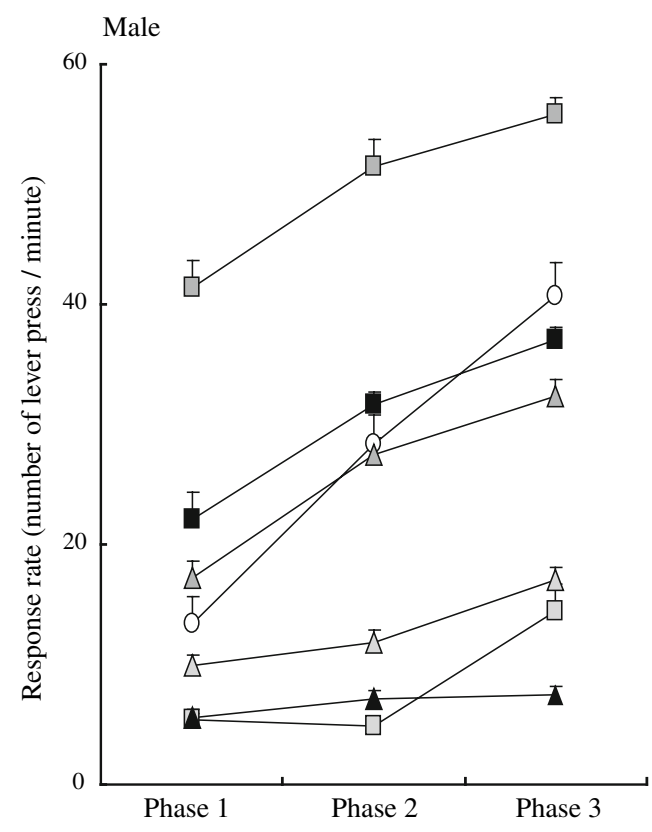

Fig. 1 Response rate (number of lever presses per minute) of male (left) and female (right) rat offspring born to dams exposed to either 2, 3, 7, 8-tetrachlorodibenzo-p-dioxin (TCDD) or 3, 3', 4, 4', 5pentachlorobiphenyl (PCB126) at three different doses in phases 1,2 and 3 in the fixed ratio (FR) component. Doses of TCDD were 50, 200 and $800 \mathrm{ng} / \mathrm{kg}$, respectively, and those of PCB126 were 500,

rates increased significantly with the advancement of the phase for both male $(p=0.005)$ and female $(p=0.005)$ rats in both the TCDD- and PCB126-exposed groups, indicating that the phase had a significant effect on the FR component. No significant effect of sex or interaction between chemical and dose on The response rate were detected in the FR component.

In the DRL component, animals in the TCDD mediumdose groups for TCDD (200 ng/ kg) and PCB126 $(2,000 \mathrm{ng} / \mathrm{kg})$ had higher response rates than animals in the other groups, albeit these were not statistically significantly different ( $p=0.068)$ (Fig. 2). Repeated measures ANOVA revealed a significant effect of phase $(p=0.0001)$ in the DRL component, and the response rate decreased with the advancement of the phase, a trend that was opposite to that in the FR component described above. No significant effect of sex on any of the indices was observed. There were also no significant chemical-dose interactions with respect to response rate in the DRL component.

Repeated measures ANOVA showed that DRL reward rate significantly increased with the advancement of the phase in both male $(p=0.0001)$ and female $(p=0.0001)$ rats (Fig. 3). No significant differences in reward rate in the DRL component were observed with respect to chemical, sex, dose, and chemical-dose in the DRL component.

The effects of phase comprised an increase in $\mathrm{RR}_{\mathrm{FR}}$, a decrease in $\mathrm{RR}_{\mathrm{DRL}}$ and an increase in $\mathrm{rR}_{\mathrm{DRL}}$ (Figs. 1-4)

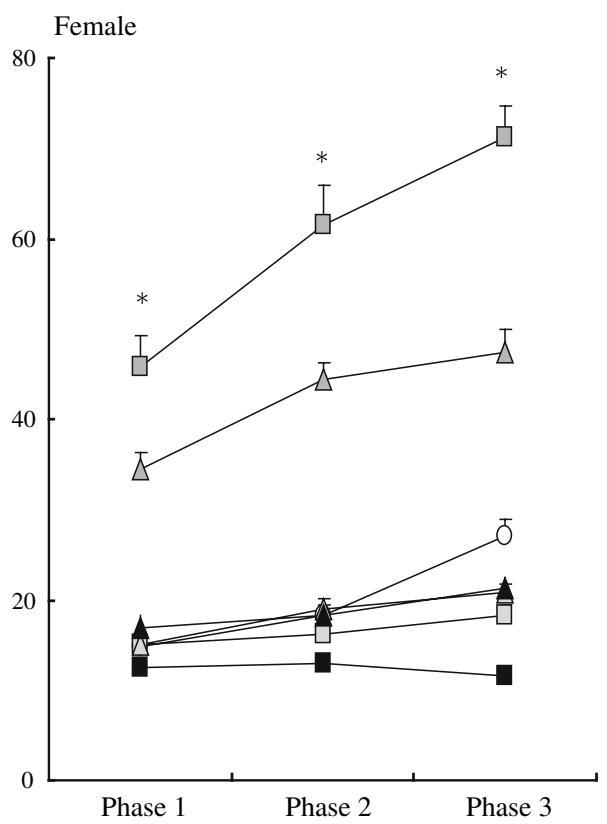

2,000 and 8,000 ng/kg, respectively. Open circle indicates control, empty, gray and black squares indicate 50, 200 and $800 \mathrm{ng} / \mathrm{kg}$ of TCDD, respectively, open, gray and black triangles indicate 500, 2,000 and $8,000 \mathrm{ng} / \mathrm{kg}$ of $\mathrm{PCB} 126$, respectively. Each symbol indicates the mean and SEM of number of lever presses in each ten-session phase. ${ }^{*} p<0.05$ (vs. other groups)

and indicated that the behavioral test protocols in our study were properly carried out during the training of the rats to acquire a reward-oriented behavior.

When the results of the learning task behavior during the last five sessions were compiled and analyzed, the mean reward rate in the FR component for male animals in the high-dose PCB126 group was significantly $(p=0.000)$ lower than that for animals in control group and in the highdose TCDD group (Fig. 5), and the reward rate of the female animals in the medium-dose TCDD group was significantly ( $p=0.000$ ) higher than that of control (Fig. 5). There were no statistical differences in the reward rates in the DRL component for both male and female groups for TCDD and PCB126 during the last five sessions (Fig. 6).

\section{Discussion}

The most important finding in our study is that both TCDD and PCB126 affected the lever-pressing response of rats in the SCOB procedure. The analysis that was performed for the response and reward rates of animals in each of the TCDD and PCB126 dose-groups indicated that the pattern of change in response or reward rates to low and medium doses was similar in the TCDD and PCB126 groups. We therefore concluded that low and medium doses of PCB126 may have effects similar to those of corresponding doses of 


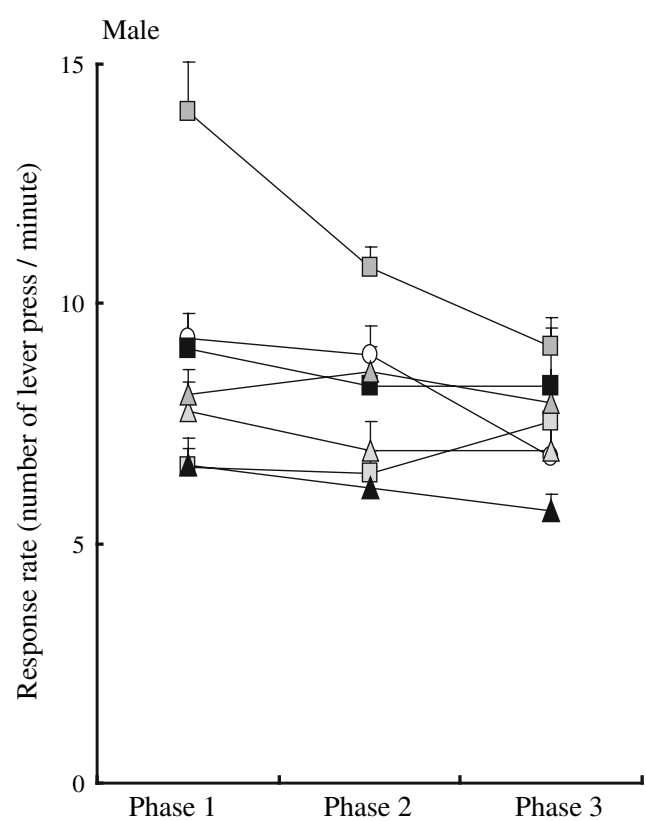

Fig. 2 Response rate (number of lever presses per minute) of male (left) and female (right) rat offspring born to dams exposed to either TCDD or PCB126 at three different doses in phases 1, 2 and 3 in the differential reinforcement of low rates (DRL) component. Open circle indicates control, empty, gray and black squares indicate 50, 200 and

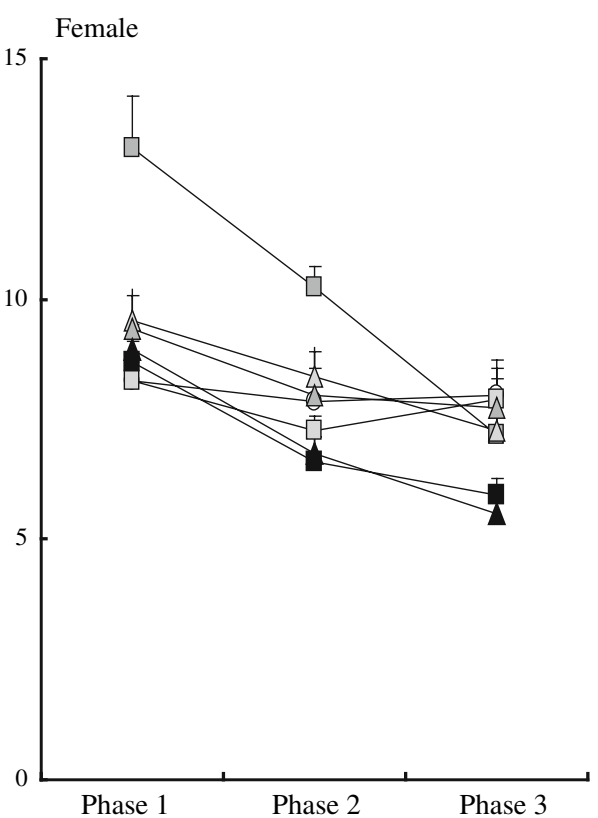

$800 \mathrm{ng} / \mathrm{kg}$ of TCDD, respectively, and open, gray and black triangles indicate 500, 2,000 and 8,000 ng/kg of PCB126, respectively. Each symbol indicates the mean and SEM of a number of lever presses in each ten-session phase. ${ }^{*} p<0.05$ (vs. other groups)

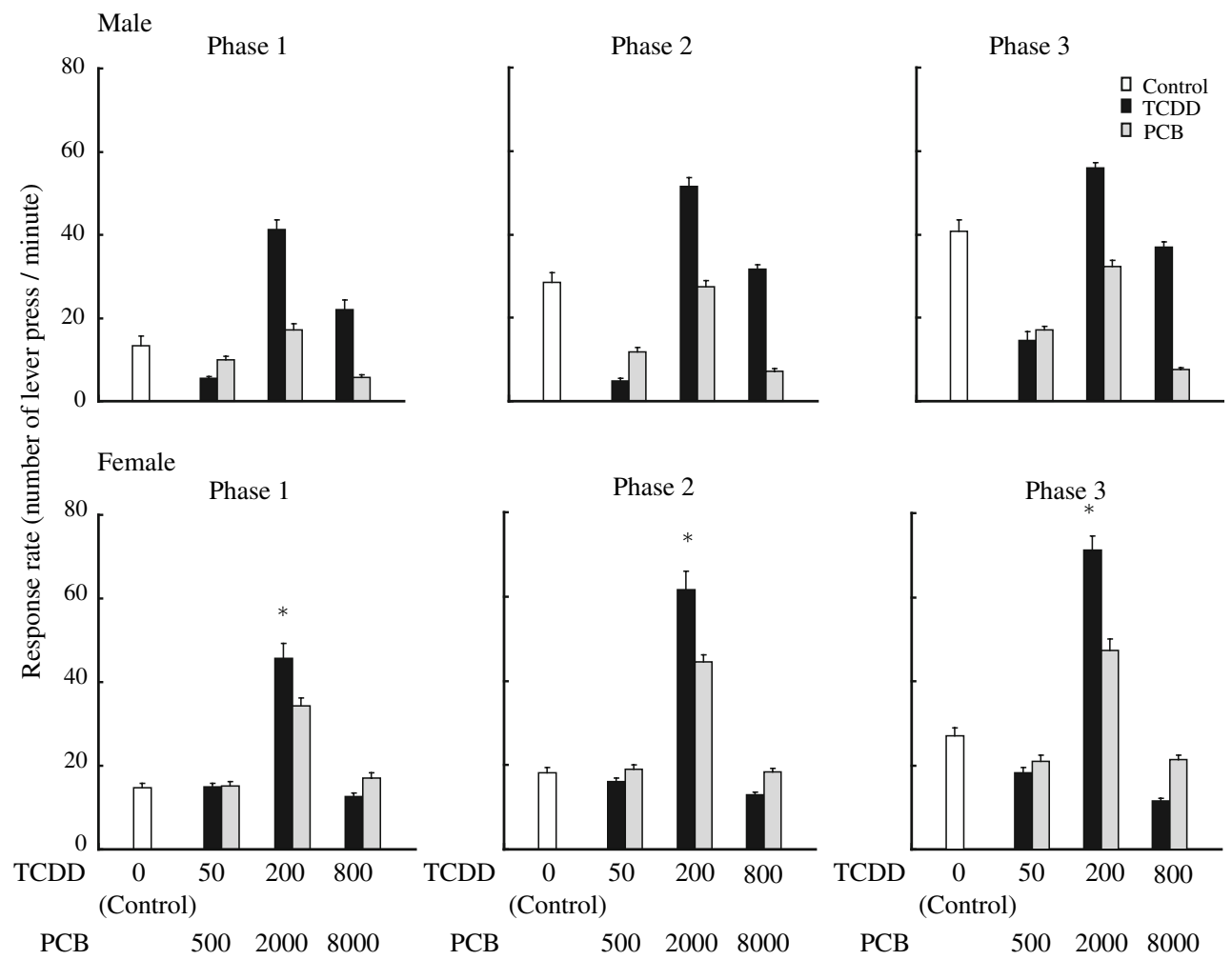

Fig. 3 Response rate (number of lever presses per minute) of male (upper) and female (lower) rat offspring born to dams exposed to either TCDD or PCB126 at three different doses in phases 1, 2 and 3 in the FR component. Doses of TCDD were 50, 200 and $800 \mathrm{ng} / \mathrm{kg}$, respectively, and those of PCB126 were 500, 2,000 and 8,000 ng/kg, respectively. Open, black and gray bars indicate control, TCDD and PCB126, respectively. Bars indicate the mean and SEM of the number of lever presses in each ten-session phase. $* p<0.05$ (vs. other groups) 
Fig. 4 Response rate (number of lever presses per minute) of male (upper) and female (lower) rat offspring born to dams exposed to either TCDD or PCB126 at three different doses in phases 1, 2 and 3 in the DRL component. Doses of TCDD were 50,200 and $800 \mathrm{ng} / \mathrm{kg}$, respectively, and those of PCB126 were 500, 2,000 and $8,000 \mathrm{ng} / \mathrm{kg}$, respectively. Open, black and gray bars indicate control, TCDD and PCB126 groups, respectively. Bars indicate the mean and SEM of the number of lever presses in each ten-session phase

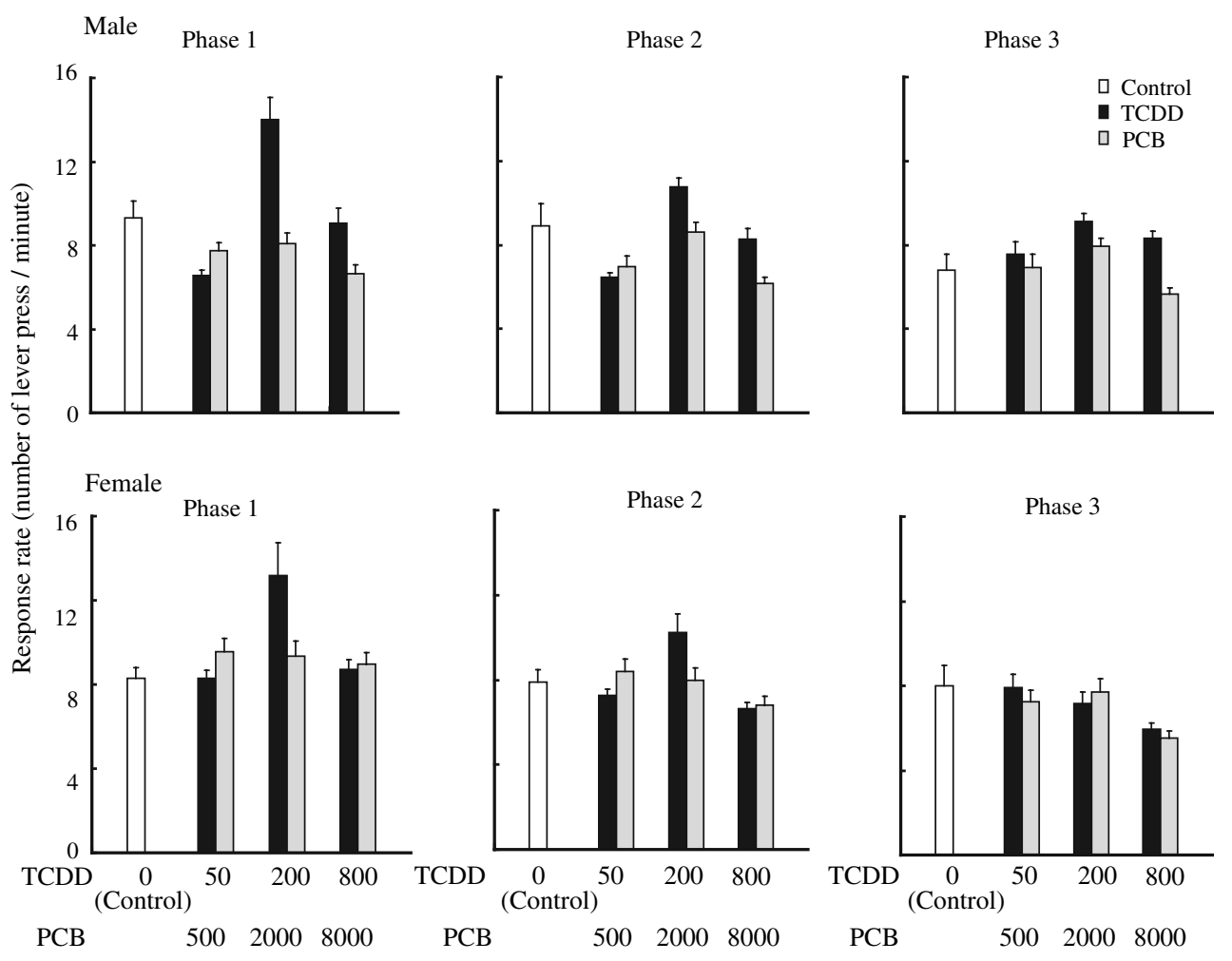

Fig. 5 Reward rate (number of food reward per minute) of male (upper) and female (lower) rat offspring born to dams exposed to either TCDD or PCB126 at three different doses in the DRL component. Doses of TCDD were 50, 200 and $800 \mathrm{ng} / \mathrm{kg}$, respectively, and those of PCB126 were 500, 2,000 and $8,000 \mathrm{ng} / \mathrm{kg}$, respectively. Open, black and gray bars indicate control, TCDD and PCB126 groups, respectively. Bars indicate the mean and SEM of the number of food reward in each ten-session phase

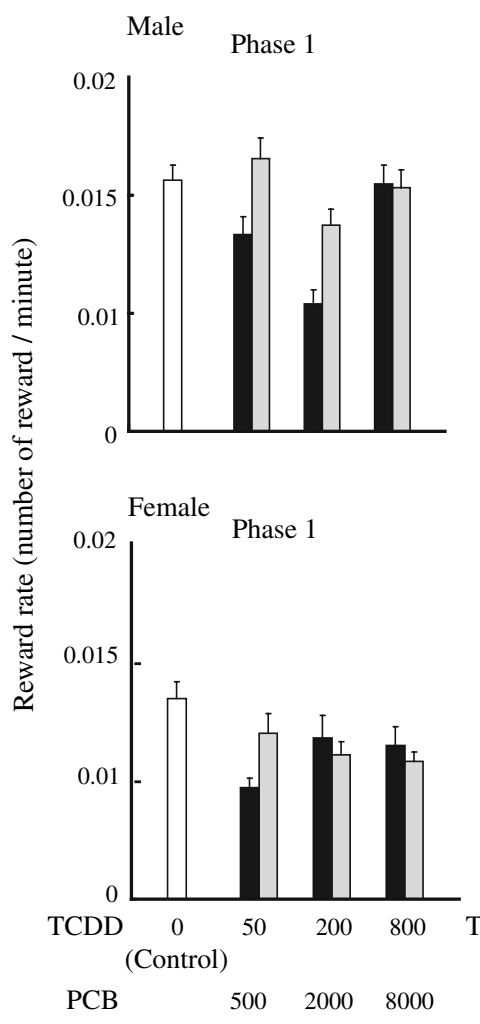

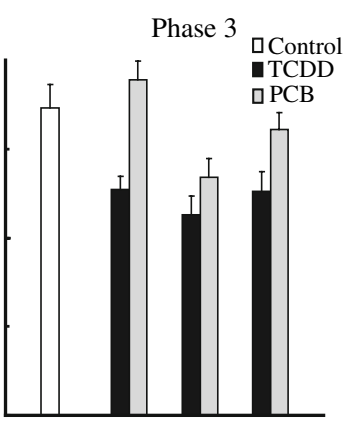
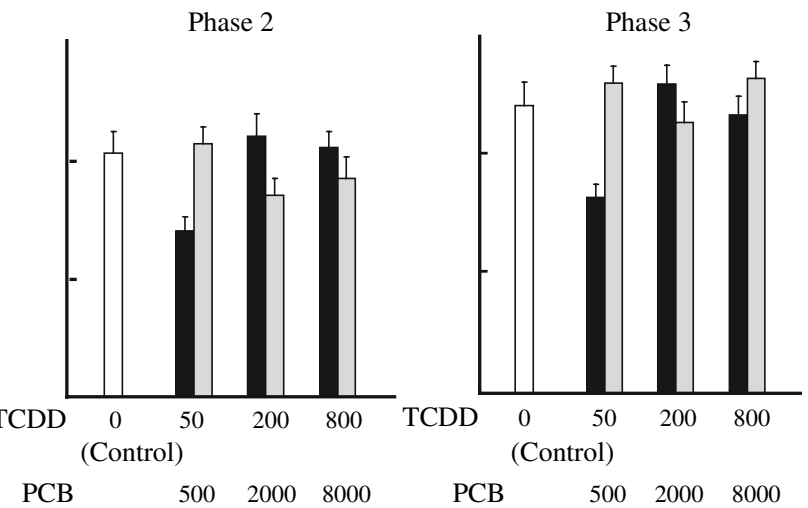

TCDD on the learning behavior of both male and female offspring. The TEF for PCB126 appropriately reflects toxic effects of PCB126. In contrast, the effect of the high dose of PCB126 was found to be different from that of the high dose of TCDD. It is possible that PCB126 has a distinct toxicity at this high level.

The results on the dose-effect relationships in $\mathrm{RR}_{\mathrm{FR}}$ and the trend of the effect in $\mathrm{RR}_{\mathrm{DRL}}$ in this study indicated that 
Fig. 6 Response rate (number of lever presses per minute) at the last five sessions in FR component for male (upper left) and female (upper right) rat offspring and in DRL

component for male (lower left) and female (lower right) in mult FR 20 DRL 20-s schedule. Open bar indicates response rate of control. The first three bars next to open bar: light gray, dark gray and black bars indicate response rates of low (50 $\mathrm{ng}$ / $\mathrm{kg})$, medium (200 ng/kg), and high $(800 \mathrm{ng} / \mathrm{kg})$ doses of TCDD, respectively, and the next three bars: light gray, dark gray and black bars indicate those of low $(500 \mathrm{ng} / \mathrm{kg})$, medium $(2,000 \mathrm{ng} / \mathrm{kg})$ and high $(8,000 \mathrm{ng} / \mathrm{kg})$ doses of PCB126, respectively. Bars indicate the mean and SEM of number of lever press per minute. ${ }^{*} p<0.05$

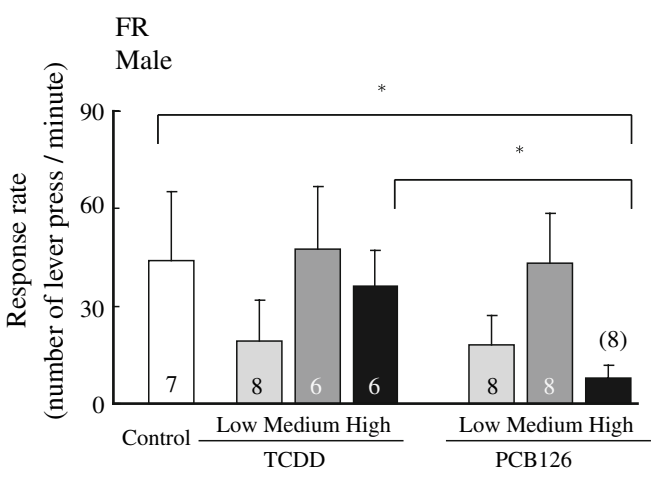

FR

Female *

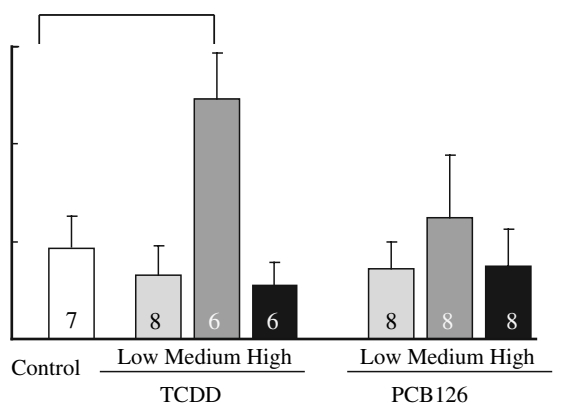

DRL

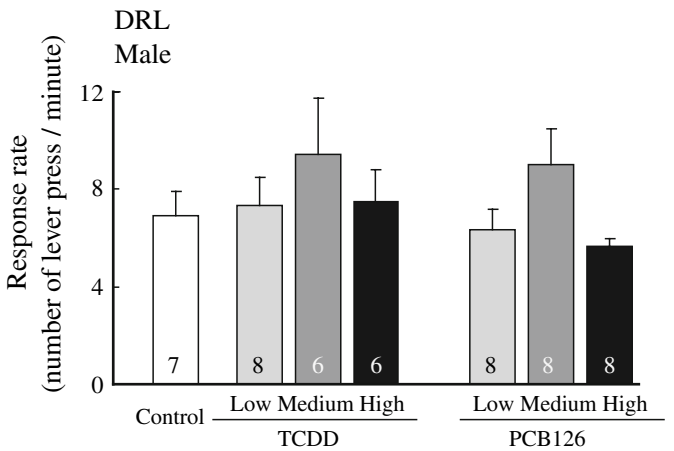

Female

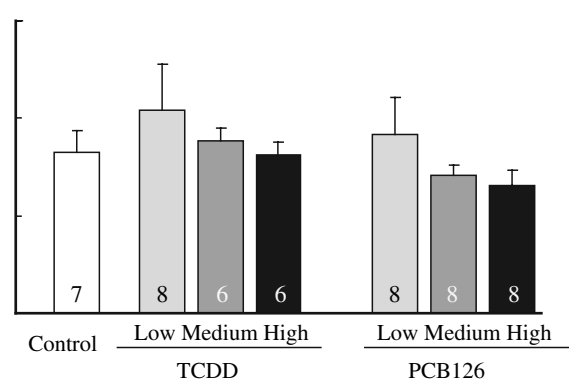

both TCDD and PCB126 affected the lever-pressing response in the SCOB procedure. The ANOVA with repeated measures analysis did not detect any significant effects of the chemicals (TCDD or PCB126) or any significant interactions between the chemical and dose (Table 3 ). In addition, the analysis indicated that the patterns of dose-effect relationships with respect to response rate for the two chemicals (TCDD and PCB126) were very similar to each other. The present results are consistent with results from previous studies in that no significant differences were found between these two chemicals in terms of two different endpoints, namely, saccharin preference and thyroxin metabolism. Amin et al. [23] examined the effects of maternal exposure to PCB126 (250 and 1,000 ng/kg) and TCDD (25 and $100 \mathrm{ng} / \mathrm{kg}$ ) on the saccharin preference of rat offspring and found that female rats consumed less saccharin and exhibited a reduced preference to saccharincontaining water following exposure to both chemicals. In terms of thyroxin metabolism, Craft et al. [24] examined serum thyroxin concentrations in adult rats exposed to

Table 3 Results of statistical analysis of response rates (numbers of response per minute) in the FR20 and DRL20s components, and reward rate (number of reward per minute) in the DRL20s component analyzed by the one-way ANOVA with repeated measures

\begin{tabular}{|c|c|c|c|c|c|c|c|}
\hline \multirow[t]{3}{*}{ Factor } & \multirow[t]{3}{*}{$d f$} & \multirow{2}{*}{\multicolumn{2}{|c|}{$\frac{\text { Fixed ratio }}{\text { Response/min }}$}} & \multicolumn{4}{|c|}{ Differential reinforcement of low rates } \\
\hline & & & & \multicolumn{2}{|c|}{ Response/min } & \multicolumn{2}{|c|}{ Reward/min } \\
\hline & & $F$ & $P$ & $F$ & $P$ & $F$ & $P$ \\
\hline Phase & 2,200 & 5.56 & 0.005 & 27.38 & 0.000 & 91.73 & 0.000 \\
\hline Chemical & 1,100 & 2.01 & 0.16 & 2.16 & 0.15 & 2.80 & 0.097 \\
\hline Dose & 3,100 & 4.41 & 0.006 & 2.45 & 0.068 & 1.59 & 0.20 \\
\hline Sex & 1,100 & 2.42 & 0.12 & 0.27 & 0.60 & 0.12 & 0.91 \\
\hline Chemical $\times$ dose & 1,100 & 1.40 & 0.25 & 0.95 & 0.42 & 1.12 & 0.35 \\
\hline Chemical $\times$ dose $\times$ phase & 1,100 & 0.40 & 0.99 & 2.10 & 0.061 & 0.93 & 0.47 \\
\hline Chemical $\times$ dose $\times$ sex & 1,100 & 0.63 & 0.60 & 0.18 & 0.91 & 0.25 & 0.86 \\
\hline
\end{tabular}

$d f$ degrees of freedom, $F$ F-value, $p$ p-value. Chemical (TCDD or PCB), dose (low, medium or high) and sex (male or female) were treated as between-subject factors, and phase (1, 2 and 3) was treated as a within-subject factor for repeated measures ANOVA. Because all of the three indices had a normal distribution, they were subjected to logarithmic transformation. Differences at $p<0.05$ were considered significant 
PCB126 $(0.03-100 \mu \mathrm{g} / \mathrm{kg})$ or TCDD $(0.003-10 \mu \mathrm{g} / \mathrm{kg})$, but these researchers found no difference in dose-dependent suppression between the two chemicals.

Animals in the medium-dose TCDD group showed significantly higher response rates than those in the other TCDD groups, while animals in the medium-dose PCB126 group showed a tendency to press the lever at a higher rate than those in the other PCB126 groups. In particular, animals in medium-dose TCDD group showed significantly higher response rates in phases 2 and 3. The medium-dose group of rats that were exposed to PCB126 or TCDD received a larger number of rewards that the rats of the other dose-groups because of an increase in response rate in the FR component. On the other hand, these groups of animals showed a tendency for an increase in the DRL component as well. Consequently, our observation of an increase in the FR component does not mean that the learning performance of the offspring born to dams exposed to these chemicals was improved. Therefore, we concluded that the animals in the medium-dose group showed hyperactive behavior rather than enhanced learning performance both in the FR and DRL components.

It is possible that the medium dose of the chemicals enhanced the activity of the rats; this explanation of the behavior may be supported by the observation that, as shown in Fig. 3, there was no significant change in the $\mathrm{rR}_{\mathrm{DRL}}$ as an index of learning function nor in that of the motor activity of animals. The apparent improvement in the spatial learning behavior of rodents exposed to either PCB126 or TCDD was also observed in a previous study [14], that is, prenatal exposure of pregnant rats to either of these compounds resulted in an apparently improved performance in their offspring; in this previous study, however, exposure to ortho-substituted PCBs $\left[2,4,4^{\prime}\right.$-TCB

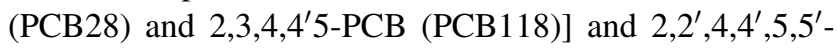
PCB (PCB153) did not change the number of errors. The interpretation of these results seems very complicated. When Schantz et al. [14] examined the spatial learning ability of pups born to and nursed by mothers exposed to either TCDD $(0.025$ or $0.1 \mu \mathrm{g} / \mathrm{kg}$ per day) or PCB126 ( 0.25 or $1.0 \mu \mathrm{g} / \mathrm{kg}$ per day) from PND 10-16, using two different spatial learning tasks, they obtained inconsistent results from these two spatial learning tasks. Although one task (radial arm maze) revealed a decrease in the number of errors in pups exposed to either TCDD or PCB126, another task (T maze) did not show any changes. This apparent discrepancy in the results is discussed from the viewpoint of a response strategy of tested rats, and the reduction in the number of errors is not considered to be due to the TCDD-induced improvement in spatial learning and memory performance [25]. Thus, the interpretation of our results remains to be confirmed in future studies.
In another study, Rice et al. [11] used a behavioral protocol in which female Long-Evans rats were given an initial loading dose of PCB 126 from 5 weeks prior to mating $(0,0.25,1.0 \mu \mathrm{g} / \mathrm{kg}$ per day) to gestation and lactation. One female and one male from each litter were selected to form groups, and the groups of animals were tested in a multiple fixed interval (FI) fixed ratio (FR) schedule of reinforcement beginning at age 200 days, followed immediately by performance on a differential reinforcement of low rates (DRL) schedule. The authors concluded that there were no effects of TCDD or PCB126 on the learning behavior of the offspring. The results of their study are inconsistent with those of our current study as well as those of other studies. In a previous study by Holene et al. [26], rats were prenatally exposed to PCB126 by gavage at $2 \mu \mathrm{g} / \mathrm{kg}$ or $5 \mathrm{mg} / \mathrm{kg}$ every second day from PNDs 3 to 13; this exposure did not have any effects on the body weight of the dams. Male offspring (16-week-old) were subjected to a reinforcement behavioral examination using a two-component (FI and extinction components) examination and were found to be hyperactive. In another study [21], pregnant Sprague-Dawley rats were orally administered a single dose of TCDD, and their male and female offspring were examined by operant behavioral examinations. The female offspring born to dams administered TCDD as low as $60 \mathrm{ng} / \mathrm{kg}$ showed an increase in response rate in an FR10 schedule. Taken together with our results, there is evidence that both TCDD and PCB126 induce hyperactivity, but this evidence does not necessarily indicate that these chemicals impair learning ability. A possible reason for this inconsistency might be attributable to differences in the duration of the exposure period and the age of the animals. In particular, the difference in the duration of the exposure periods between previous studies and our study may be critical. We have shown here, for the first time, the acute toxic effects of an ultra-low dose of these chemicals.

There are two possible reasons for the inconsistency between these two previous studies and our study. First, Rice et al. [11] used a same strain of rats and carried out their tests using a similar SCOB procedure as we did, but the exposure period and the age of the animals differ from those in our study. In particular, the length of the exposure period in their studies was longer than that of our study; as such, the accumulated amount of PCB126 in the animals might have induced the same effects as those shown in the high-dose PCB126 group in our study, in which the response rates of the animals in both FR and DRL components were not different from those of the control group.

In contrast, Holene et al. [26] reported that male offspring rats (16-week-old), prenatally exposed to $2 \mu \mathrm{g} / \mathrm{kg}$ or $5 \mathrm{mg} / \mathrm{kg}$ of PCB126 every second day from PNDs 3 to 13 , were found to be hyperactive in an operant test using a 
two-component test (FI and extinction components). In another study [21], pregnant Sprague-Dawley rats were orally administered a single dose of TCDD, and their male and female offspring were examined by operant behavioral examinations. The female offspring born to dams administered TCDD as low as $60 \mathrm{ng} / \mathrm{kg}$ showed an increase in response rate in an FR10 schedule. Taken together, our current results provide experimental evidence that both TCDD and PCB126 induce hyperactivity, but they do not necessarily indicate they impair learning ability.

Analysis during the last five sessions as a learning steady state revealed a significant difference between the effects of the two chemicals at the highest doses, particularly in male offspring, and it is reasonable to speculate that dioxin-like PCBs, including PCB126, have as yet unidentified AhR-independent effects, albeit the fact that the degree of independency from AhR may vary significantly among dioxin-like PCB congeners. The toxic effect of the highest dose of PCB126 on the learning task that we detected was clarified only when the learning behavior reached the steady state during the last part of the SCOB procedure, and it affected only male animals. The results suggest that the toxicity specific to PCB126 might be expressed in specific learning circumstances, such as the steady state, or it may be sex-linked. Furthermore, non-dioxin-like PCBs, such as PCB153, have some significant neurotoxicological effects on learning behavior in rodent offspring [27]. Thus, further studies are needed to clarify the mechanism underlying the AhR-independent effects of PCBs on neurobehavioral functions for the use of risk assessment of these compounds.

In conclusion, maternal exposure to TCDD and PCB126 affected the neurobehavioral performance of rat offspring in the SCOB procedure with a similar dose-response pattern, which supports the current TEF value of 0.1 for PCB126 derived from REP values obtained from other experimental data in vivo and in vitro.

Acknowledgments We thank S. Hori, M. Mano and M. Suzuki for their excellent technical assistance. This study is supported in part by the Ministry of the Environment of Japan (to C. Tohyama) and in part by a grant from the National Institute for Environmental Studies in Japan.

\section{References}

1. Van den Berg M, Birnbaum LS, Denison M, De Vito M, Farland W, Feeley M, et al. The 2005 World Health Organization reevaluation of human and mammalian toxic equivalency factors for dioxins and dioxin-like compounds. Toxicol Sci. 2006;93:223-41.

2. Hooper K, Clark GC. Workshop on perinatal exposure to dioxinlike compounds. VI. Role of biomarkers. Environ Health Perspect. 1995; Suppl 2:161-7.
3. Van den Berg M, Birnbaum L, Bosveld AT, Brunstrom B, Cook $\mathrm{P}$, Feeley M, et al. Toxic equivalency factors (TEFs) for PCBs, PCDDs, PCDFs for humans and wildlife. Environ Health Perspect. 1998;106:775-92.

4. Ahlborg UG, Brouwer A, Fingerhut MA, Jacobson JL, Jacobson SW, Kennedy SW, et al. Impact of polychlorinated dibenzo- $p$ dioxins, dibenzofurans, and biphenyls on human and environmental health, with special emphasis on application of the toxic equivalency factor concept. Eur J Pharmacol. 1992;228:179-99.

5. Birnbaum LS, DeVito MJ. Use of toxic equivalency factors for risk assessment for dioxins and related compounds. Toxicology. 1995;105:391-401.

6. Haws LC, Su SH, Harris M, Devito MJ, Walker NJ, Farland WH, et al. Development of a refined database of mammalian relative potency estimates for dioxin-like compounds. Toxicol Sci. 2006;89:4-30.

7. Hojo R, Kakeyama M, Yonemoto J, Tohyama C. Perinatal exposure to dioxins perturbs learning performance of the rat in a dose-specific fashion. Organohalogen Compd. 2004;66:3143-7.

8. Bushnell PJ, Rice DC. Behavioral assessments of learning and attention in rats exposed perinatally to $3,3^{\prime}, 4,4^{\prime}, 5$-pentachlorobiphenyl (PCB 126). Neurotoxicol Teratol. 1999;21:381-92.

9. Holene E, Nafstad I, Skaare JU, Sagvolden T. Behavioural hyperactivity in rats following postnatal exposure to sub-toxic doses of polychlorinated biphenyl congeners 153 and 126. Behav Brain Res. 1998;94:213-24.

10. Rice DC. Effect of exposure to $3,3^{\prime}, 4,4^{\prime}, 5$-pentachlorobiphenyl (PCB 126) throughout gestation and lactation on development and spatial delayed alternation performance in rats. Neurotoxicol Teratol. 1999;21:59-69.

11. Rice DC, Hayward S. Lack of effect of 3,3'4,4',5-pentachlorobiphenyl (PCB 126) throughout gestation and lactation on multiple fixed interval-fixed ratio and DRL performance in rats. Neurotoxicol Teratol. 1998;20:645-50.

12. Rice DC, Hayward S. Effects of exposure to $3,3^{\prime}, 4,4^{\prime}, 5$-pentachlorobiphenyl (PCB 126) throughout gestation and lactation on behavior (concurrent random interval-random interval and progressive ratio performance) in rats. Neurotoxicol Teratol. 1999;21:679-87.

13. Seo BW, Sparks AJ, Medora K, Amin S, Schantz SL. Learning and memory in rats gestationally and lactationally exposed to 2,3,7,8-tetrachlorodibenzo- $p$-dioxin (TCDD). Neurotoxicol Teratol. 1999;21:231-9.

14. Schantz SL, Seo BW, Moshtaghian J, Peterson RE, Moore RW. Effects of gestational and lactational exposure to TCDD or coplanar PCBs on spatial learning. Neurotoxicol Teratol. 1996;18:305-13.

15. Gomita Y, Ichimaru Y, Moriyama M, Araki H, Futagami K. Effects of anxiolytic drugs on rewarding and aversive behaviors induced by intracranial stimulation. Acta Med Okayama. 2003;57:95-108.

16. Hudzik TJ, Yanek M, Porrey T, Evenden J, Paronis C, Mastrangelo $\mathrm{M}$, et al. Behavioral pharmacology of AR-A000002, a novel, selective 5-hydroxytryptamine(1B) antagonist. J Pharmacol Exp Ther. 2003;304:1072-84.

17. Laties VG. How operant conditioning can contribute to behavioral toxicology. Environ Health Perspect. 1978;26:29-35.

18. Staddon JE, Cerutti DT. Operant conditioning. Annu Rev Psychol. 2003;54:115-44.

19. Schantz SL, Bowman RE. Learning in monkeys exposed perinatally to 2,3,7,8-tetrachlorodibenzo-p-dioxin (TCDD). Neurotoxicol Teratol. 1989;11:13-9.

20. Markowski VP, Cox C, Preston R, Weiss B. Impaired cued delayed alternation behavior in adult rat offspring following exposure to 2,3,7,8-tetrachlorodibenzo- $p$-dioxin on gestation day 15. Neurotoxicol Teratol. 2002;24:209-18. 
21. Hojo R, Stern S, Zareba G, Markowski VP, Cox C, Kost JT, et al. Sexually dimorphic behavioral responses to prenatal dioxin exposure. Environ Health Perspect. 2002;110:247-54.

22. Peterson RE, Theobald HM, Kimmel GL. Developmental and reproductive toxicity of dioxins and related compounds: crossspecies comparisons. Crit Rev Toxicol. 1993;23:283-335.

23. Amin S, Moore RW, Peterson RE, Schantz SL. Gestational and lactational exposure to TCDD or coplanar PCBs alters adult expression of saccharin preference behavior in female rats. Neurotoxicol Teratol. 2000;22:675-82.

24. Craft ES, DeVito MJ, Crofton KM. Comparative responsiveness of hypothyroxinemia and hepatic enzyme induction in LongEvans rats versus C57BL/6J mice exposed to TCDD-like and phenobarbital-like polychlorinated biphenyl congeners. Toxicol Sci. 2002;68:372-80.
25. Teobald HM, Kimmel GL, Peterson RE. Developmental and reproductive toxicity of dioxins and related chemicals. In: Schecter A, Gasiewicz TA, editors. Dioxins and health. 2nd edn. Hobeken: Wiley; 2003. pp. 329-432.

26. Holene E, Nafstad I, Skaare JU, Krogh H, Sagvolden T. Behavioural effects in female rats of postnatal exposure to sub-toxic doses of polychlorinated biphenyl congener 153. Acta Paediatr Suppl. 1999;88:55-63.

27. Hojo R, Kakeyama M, Yonemoto J, Tohyama C. Gestational exposure to $2,2^{\prime}, 4,4^{\prime}, 5,5^{\prime}$-hexachlorobiphenyl(PCB153) impairs learning performance in rat offspring. Organohalogen Compd. $2005 ; 67: 2394-5$. 